IMPERIAL/TP/94-95/11

TUTP-95-2

NI94039

hep-ph/9501266

\title{
Phase equilibration in bubble collisions
}

\author{
T.W.B. Kibble \\ Blackett Laboratory, Imperial College, London SW7 2BZ, United Kingdom and \\ Isaac Newton Institute for Mathematical Sciences, Cambridge CB3 OEH, United Kingdom \\ Alexander Vilenkin \\ Institute of Cosmology, Department of Physics and Astronomy, \\ Tufts University, Medford MA 02155, USA and \\ Isaac Newton Institute for Mathematical Sciences, Cambridge CB3 OEH, United Kingdom
}

()

\begin{abstract}
In the context of an Abelian gauge symmetry, spontaneously broken at a first-order transition, we discuss the evolution of the phase difference between the Higgs fields in colliding bubbles. We show that the effect of dissipation, represented by a finite plasma conductivity, is to cause the phases to equlibrate on a time-scale, determined by the conductivity, which can be much smaller than the bubble radii at the time of collision. Currents induced during the phase equilibration generate a magnetic flux, which is determined by the initial phase difference. In a three-bubble collision, the fluxes produced by each pair of bubbles combine, and a vortex can be formed. We find that, under most conditions, the probability of trapping magnetic flux to form a vortex is correctly given by the "geodesic rule".
\end{abstract}

98.80. $\mathrm{Cq}$ 


\section{INTRODUCTION}

Phase transitions in the early universe, as in condensed-matter systems, may lead to the formation of defects, such as cosmic strings [1]. To determine the observational implications of defects one must follow their evolution from an initial state soon after the phase transition up to the time when they begin to have visible effects. There has recently been renewed interest in the problem of estimating the initial defect density, which provides the starting point for such studies.

In this paper, we are concerned with the initial number density of strings formed at a first-order phase transition proceeding by bubble nucleation. For simplicity, we consider an Abelian theory with a scalar Higgs field $\Phi$, described by the Lagrangian

$$
\mathcal{L}=D_{\mu} \Phi^{*} D^{\mu} \Phi-\frac{1}{4} F_{\mu \nu} F^{\mu \nu}-V(\Phi),
$$

where $D_{\mu} \Phi=\partial_{\mu} \Phi+i e A_{\mu} \Phi, F_{\mu \nu}=\partial_{\mu} A_{\nu}-\partial_{\nu} A_{\mu}$, and the potential $V$ is a function of $|\Phi|$ with a local minimum at $|\Phi|=0$ and a global minimum at $|\Phi|=\eta / \sqrt{ } 2$.

Nothing happens at the theoretical critical temperature $T_{\mathrm{c}}$ (where the two minima of the high-temperature effective potential are degenerate). Once the temperature has fallen well below $T_{\mathrm{c}}$, tunnelling can occur from the false vacuum state at $\Phi=0$ to the true vacuum, where $\Phi \approx \eta e^{i \theta} / \sqrt{ } 2$. This is an example of spontaneous symmetry breaking: within each bubble there is a random choice of the phase angle $\theta$.

The bubbles nucleate at random points in space and then expand. The bubble walls accelerate, often nearly reaching the speed of light, until they meet and fill the whole of space. The nucleation rate per unit space-time volume is determined by the tunnelling probability, which may be calculated from the "bounce" solution of the Euclidean field theory [2]. It is likely to be a rapidly rising function of time, but for purposes of illustration let us assume that it is zero up to some given time and thereafter a constant $\gamma$. We also suppose that the bubble walls expand at speed $v$, with $\gamma v^{3} \gg H^{4}$ (where $H$ is the Hubble parameter). Then the final mean number density of bubbles will be approximately $1 / \xi^{3}$, where $\xi=(v / \gamma)^{1 / 4}$. (These assumptions are in no way critical to our discussion.)

The traditional picture of string formation is as follows [3]. Within each bubble the phase $\theta$ is essentially constant, but phases in different bubbles are uncorrelated. When two bubbles with phases $\theta_{1}$ and $\theta_{2}$ meet, the sharp discontinuity in the phase in smoothed out to become a smooth variation. For energetic reasons, the system will tend to choose the shorter of the two paths between $\theta_{1}$ and $\theta_{2}$. For example, if $\theta_{1}=0$ and $0<\theta_{2}<\pi$, the phase will increase from 0 to $\theta_{2}$; but if $\pi<\theta_{2}<2 \pi$, it will instead decrease from 0 to $\theta_{2}-2 \pi$. This is often called the "geodesic rule". It has an obvious extension to higher symmetry groups.

When three bubbles meet, a string or vortex may be trapped in the region between them. Whether this happens or not is determined by the net phase change in going from $\theta_{1}$ to $\theta_{2}$, to $\theta_{3}$ and back to $\theta_{1}$. It is easy to see that if the three angles are independent random variables, uniformly distributed between 0 and $2 \pi$, and if the geodesic rule is correct, then the probability of trapping a string is $1 / 4$. Thus the initial string density (length per unit volume) is of order $1 / 4 \xi^{2}$.

This is obviously only a crude estimate. Several of the assumptions we have made are questionable. Some of the objections are relatively minor. It is clearly not precisely true that the phase within each bubble is constant. There must be random thermal and quantum 
fluctuations. So the geodesic rule will sometimes break down. When two bubbles meet, there may be local random fluctuations that will cause the phase to go the "wrong" way round in joining $\theta_{1}$ and $\theta_{2}$. But the effect of this would merely be a slight increase in the probability of trapping a string. Indeed, there could also be a nonzero probability that the net phase change would be $\pm 4 \pi$, thus trapping a string of winding number \pm 2 .

As the bubbles grow, closed loops within which strings may be trapped will often be formed of more than three bubbles. However, as the bubbles expand further into the trapped region, it will generally be split up into several smaller regions. Eventually, the remaining gaps will always be finally closed by the junction of three bubbles.

There is a particular problem in cases where the velocity of the bubble walls is low. When $v \approx c$ we can be assured that no bubble collision can be causally affected by any previous collision (because any two points on the expanding light cone are spacelike separated). But when $v \ll c$, phase equilibration within a composite bubble might have been completed before the two components encounter a third bubble, thus reducing the chances of trapping a string. Of course, some strings would still be trapped, because the three collisions would sometimes occur nearly simultaneously, but the string density would certainly be less. One very interesting open question (to which we hope to return) is whether in that event the relative proportion of small loops to long strings would be changed. At first sight one might think that if strings are rare then most of them would form small loops [4]. If so, that would have a dramatic impact on the resulting cosmology.

Underlying all of these points is a more serious concern: does it make sense to talk of phase differences between bubbles in a gauge theory? Indeed by a gauge transformation, the phase difference can be set equal to any value we please. It has been argued by Rudaz and Srivastava [5] that string formation in gauge theories might be strongly suppressed, although a recent analysis by Hindmarsh et al [6] suggests that it is not.

It is clearly important to understand the process of string formation in gauge theories. We begin by studying what happens when two bubbles meet. An important feature of the process of phase equilibration (not included in previous analyses) is the role of dissipation. We include an ohmic dissipation term in our equations. However, it will be useful first to consider the problem in the absence of dissipation, especially to clarify some issues relating to gauge choice and boundary conditions.

There are two ways of dealing with the problem of gauge invariance. One is to use only gauge-invariant quantities, such as the covariant derivative $D_{\mu} \theta=\partial_{\mu} \theta+e A_{\mu}$. This approach provides valuable insights, but it is difficult to apply to the string-formation problem because we have to consider non-local variables such as the line integral of $D_{\mu} \theta$. The other is to choose a gauge, but that too has its problems. We need a complete gauge choice, not merely a class of gauges like the Lorentz gauge within which some gauge freedom still exists.

There are several gauges we might choose:

I. The Coulomb gauge. This works well provided we are considering only a limited region, outside of which there are no relevant charges. It is problematic in other cases, because boundary conditions are needed to solve the Poisson equation for the scalar potential $\phi \equiv A^{0}$. In practice, this gauge is not as convenient as the others, so we shall not consider it further.

II. The unitary gauge, defined by setting the phase angle $\theta$ to zero. This is very convenient within any simply connected region where $\Phi \neq 0$. Unfortunately, it does not work if the 
region is non-simply connected or contains zeroes of $\Phi$, i.e., when strings are present. It will be useful in discussing the collision of two bubbles, but is less easy to use to study three-bubble collisions.

III. The axial gauge defined by $A^{z}=0$, together with a choice of gauge on the surface $z=0$. This is particularly well suited to the problem of two colliding bubbles, where it matches the symmetry of the problem and provides a very neat solution, as we shall see in the next section. However, it is not very convenient for more general problems.

IV. The temporal gauge, given by $A^{0}=0$. This is universally applicable, and very simply related to the unitary gauge. To define it completely, we need a gauge choice at some initial time, but in our case this is straightforward: we assume that no electromagnetic fields are present before bubble nucleation starts, so that we can simply set $\mathbf{A}=\mathbf{0}$ at that time. The gauge is then defined for all times. However, this gauge does introduce somewhat artificial spatial discontinuities in various functions.

It will be useful first to examin the collision of two bubbles with no damping of the phase oscillations. Although this is a rather artificial problem, the results will help to motivate some of the more general discussion later. We begin by defining the gauge-invariant phase difference which plays an important role in determining the outcome of the collision.

\section{GAUGE-INVARIANT PHASE DIFFERENCE}

It is convenient to write the Higgs field in polar form:

$$
\Phi=\frac{1}{\sqrt{ } 2} X e^{i \theta}
$$

Then the equation of motion for $\theta$ is simply the continuity equation $\partial_{\mu} j^{\mu}=0$ for the current

$$
j_{\mu}=-e X^{2} D_{\mu} \theta \equiv-e X^{2}\left(\partial_{\mu} \theta+e A_{\mu}\right) .
$$

The phase gradient $D_{\mu} \theta$ is gauge-invariant and can be used to define a gauge-invariant phase difference between the two bubbles,

$$
\Delta \theta=\int_{A}^{B} d x^{k} D_{k} \theta
$$

where $k=1,2,3$ and points $A$ and $B$ are taken in bubble interiors. While the bubbles are well-separated, $D_{\mu} \theta$ is appreciably different from zero only in the region where $X \approx 0$, so that $j_{\mu} \approx 0$ and $F_{\mu \nu} \approx 0$ (assuming that $F_{\mu \nu}=0$ prior to bubble nucleation). Then $\Delta \theta$ is independent of the choice of path between $A$ and $B$. In general of course, $\Delta \theta$ is pathdependent, and to make it well-defined, we shall choose the integration path to be along the straight line passing through the bubble centers.

To analyse what happens when the bubbles collide, we shall assume that the radial mode is strongly damped, so that $X$ settles rapidly to its equilibrium value $\eta$. The equation for $\theta$ is then the Klein-Gordon equation

$$
\left(\partial^{2}+m_{A}^{2}\right) D_{\mu} \theta=0
$$

with the gauge boson mass $m_{A}=e \eta$. 
Let us first consider the central region of the collision, where the bubble walls can be well approximated as parallel approaching planes, so that the problem becomes one-dimensional. The initial configuration, just after the bubbles have collided, say at $z=0$ and $t=0$, will then have $D_{x} \theta=D_{y} \theta=0$ and $D_{z} \theta, D_{t} \theta$ concentrated near $z=0$ within some distance $\delta$. If the energy of the colliding walls is "instantly" dissipated, then $\delta$ is comparable to the Lorentz-contracted wall thickness. (However, if the motion of the walls is relativistic, they are likely to overshoot and go through several oscillations before loosing their energy [7]. In this case the collision region will be much wider and the phase equilibration process may be more complicated).

It is not difficult to understand that in the course of the following evolution the "wave packets" of $D_{z} \theta$ and $D_{t} \theta$ will spread in the $z$-direction and oscillate at the frequency $\omega=m_{A}$. The speed of spreading, $u$, will be determined by the initial width of the packets: $u \sim 1 / m_{A} \delta$ for $\delta \gg m_{A}^{-1}$ and $u \sim 1$ for $\delta \lesssim m_{A}^{-1}$. (This qualitative picture is confirmed by explicit solutions of Eq.(5) with Gaussian "packets").

An equation for the phase difference

$$
\Delta \theta=\int_{-z_{0}}^{z_{0}} d z D_{z} \theta
$$

can be obtained by integrating Eq.(5) over $z$,

$$
\left(\frac{d^{2}}{d t^{2}}+m_{A}^{2}\right) \Delta \theta=0
$$

The boundary terms vanish as long as $z_{0} \gg u t$. The solution of (7) is $\Delta \theta \propto \cos \left(m_{A} t\right)$. We note the "acausal" behavior of $\Delta \theta$ : it varies on a microscopic timescale $\sim m_{A}^{-1}$ which can be much smaller than the radii of the colliding bubbles.

To extend the analysis beyond the central collision region, we shall now fix the gauge and solve the field equations for $\theta$ and $A_{\mu}$. Different gauges provide useful, and complementary, insights.

\section{COLLISIONS WITHOUT DISSIPATION}

We consider the simplest possible case, where the bubble walls are assumed to move essentially with the speed of light. Without loss of generality, we can choose a frame of reference in which the two bubbles nucleate simultaneously, say at the points $(0,0,0, \pm R)$. The bubbles first collide at $(R, 0,0,0)$, when the radii are $R$. The problem has a high degree of symmetry: it is invariant under the 3 -dimensional Lorentz group $\mathrm{SO}(1,2)$ in the $(t, x, y)$ subspace [2, []. The bubble collision occurs along the surface $z=0, t^{2}-x^{2}-y^{2}=R^{2}$; for any point on that surface there is a frame of reference in which that is the point of first contact.

The obvious gauge to use here is the axial gauge which picks out the one distinguished direction, the vector joining the nucleation centres. The symmetry of the problem shows that in this gauge we must have

$$
\theta(x)=\theta(\tau, z), \quad A^{\alpha}(x)=x^{\alpha} a(\tau, z),
$$


where $\alpha=(0,1,2)$ and

$$
\tau^{2}=x^{\alpha} x_{\alpha}=t^{2}-x^{2}-y^{2} .
$$

The $z$ component of the Maxwell field equation, with the current (3), yields (with a suitable choice of arbitrary constant),

$$
\theta(\tau, z)=\frac{1}{e \eta^{2}}\left(3 a+\tau \partial_{\tau} a\right) .
$$

Thus there is just one independent unkown function.

The remaining Maxwell equations, together with the continuity equation for the current, show at once that both $\theta$ and $A^{\alpha}$ obey the Klein-Gordon equation with mass $e \eta$, whence the functions $\theta$ and $a$ satisfy the equations

$$
\begin{aligned}
& \partial_{\tau}^{2} \theta+\frac{2}{\tau} \partial_{\tau} \theta-\partial_{z}^{2} \theta+e^{2} \eta^{2} \theta=0, \\
& \partial_{\tau}^{2} a+\frac{4}{\tau} \partial_{\tau} a-\partial_{z}^{2} a+e^{2} \eta^{2} a=0 .
\end{aligned}
$$

For the initial conditions we assume that as we go from one bubble into the other, $\theta$ changes rapidly, say from $-\theta_{0}$ to $\theta_{0}$, while $\dot{\theta}=0$. Thus

$$
\left.\theta\right|_{\tau=R}=\theta_{0} \epsilon(z),\left.\quad \partial_{\tau} \theta\right|_{\tau=R}=0 .
$$

We also require that $A^{\alpha}(x)=0$ up to and on the boundary of the bubble overlap region. Using (12) and (10), this yields

$$
\left.a\right|_{\tau=R}=0,\left.\quad \partial_{\tau} a\right|_{\tau=R}=\frac{e \eta^{2}}{R} \theta_{0} \epsilon(z) .
$$

It is straightforward to solve these equations. The solution for $\theta_{\mathrm{a}}$ (the subscript indicates the gauge choice) is

$$
\theta_{\mathrm{a}}=\frac{\theta_{0} R}{\pi \tau} \int \frac{d k}{k} \sin k z\left(\cos \omega(\tau-R)+\frac{1}{\omega R} \sin \omega(\tau-R)\right),
$$

where

$$
\omega^{2}=\mathbf{k}^{2}+e^{2} \eta^{2}
$$

(It is also possible to write this explicitly in terms of the Bessel function of order zero, $\left.J_{0}\left(e \eta \sqrt{(\tau-R)^{2}-z^{2}}\right).\right)$

The gauge-invariant phase difference $\Delta \theta$ can now be deduced from the asymptotic behavior of $\theta_{\mathrm{a}}(t, x, y, z)$ at $z \rightarrow \pm \infty$,

$$
\Delta \theta(t)=\theta_{\mathrm{a}}(t, 0,0,+\infty)-\theta_{\mathrm{a}}(t, 0,0,-\infty) .
$$

This gives 


$$
\Delta \theta=\frac{2 R}{t} \theta_{0}\left(\cos e \eta(t-R)+\frac{1}{e \eta R} \sin e \eta(t-R)\right) .
$$

Thus phase equilibration does occur in this gauge with a time scale determined, as one might expect, by the size $R$ of the colliding bubbles, with superimposed oscillations with frequency given by the gauge-field mass.

Similarly, $a_{\mathrm{a}}$ is given by

$$
a_{\mathrm{a}}=\frac{\theta_{0} e \eta^{2}}{\pi \tau^{3}} \int \frac{d k}{k} \sin k z\left[-\frac{\tau-R}{\omega^{2} R} \cos \omega(\tau-R)+\left(\frac{\tau}{\omega}+\frac{1}{\omega^{3} R}\right) \sin \omega(\tau-R)\right] .
$$

It is of course straightforward to transform to other gauges. The unitary-gauge fields are given by $A_{\mathrm{u}}^{\mu}=A_{\mathrm{a}}^{\mu}+(1 / e) \partial^{\mu} \theta_{\mathrm{a}}$. These fields still exhibit the full symmetry of the problem; one finds

$$
A_{\mathrm{u}}^{\alpha}=x^{\alpha} b(\tau, z), \quad b(\tau, z)=\frac{1}{e^{2} \eta^{2}} \partial_{z}^{2} a(\tau, z)
$$

and

$$
A_{\mathrm{u}}^{z}=-\frac{1}{e} \partial_{z} \theta_{\mathrm{a}}(\tau, z)
$$

The unitary-gauge fields again obey the Klein-Gordon equation together with the initial conditions

$$
\begin{gathered}
\left.A_{\mathrm{u}}^{\alpha}\right|_{\tau=R}=0,\left.\quad \partial_{\beta} A_{\mathrm{u}}^{\alpha}\right|_{\tau=R}=\frac{x^{\alpha} x_{\beta}}{R^{2}} \frac{2 \theta_{0}}{e} \delta^{\prime}(z), \\
\left.A_{\mathrm{u}}^{z}\right|_{\tau=R}=-\frac{2 \theta_{0}}{e} \delta(z),\left.\quad \partial_{\alpha} A_{\mathrm{u}}^{z}\right|_{\tau=R}=0 .
\end{gathered}
$$

Finally, we can transform to the temporal gauge which, as we shall see, has some rather odd features. We find $\theta_{\mathrm{t}}$ by integrating the equation

$$
\dot{\theta}_{\mathrm{t}}=e A_{\mathrm{u}}^{0} \text {. }
$$

We need an initial condition. Assuming as before that outside the bubble overlap region $\theta=\theta_{0} \epsilon(z)$, and using (19), we find

$$
\theta_{\mathrm{t}}=\theta_{0} \epsilon(z)+e \int_{R}^{\tau} \tau b d \tau=\theta_{\mathrm{a}}+e \int_{R}^{\tau} \tau a d \tau
$$

It is interesting to note that in spite of our non-covariant choice of gauge, $\theta_{\mathrm{t}}$ is still a function only of $\tau$ and $z$. It satisfes exactly the same initial conditions (12) as $\theta_{\mathrm{a}}$. However it does not satisfy the Klein-Gordon equation. If we integrate the $\mu=0$ Maxwell equation (the Gauss law) using the given initial conditions, we find

$$
\partial_{k} A_{\mathrm{t}}^{k}=e \eta^{2}\left[\theta_{\mathrm{t}}-\theta_{0} \epsilon(z)\right] .
$$

Thus $\theta_{\mathrm{t}}$ actually obeys the equation 


$$
\partial^{2} \theta_{\mathrm{t}}+e^{2} \eta^{2}\left[\theta_{\mathrm{t}}-\theta_{0} \epsilon(z)\right]=0
$$

The symmetry of the problem also shows up, even more remarkably perhaps, in the fact that not only does $A_{\mathrm{t}}^{0}=0$ but in fact $A_{\mathrm{t}}^{\alpha}=0$. The only non-zero component of the gauge potential is

$$
A_{\mathrm{t}}^{z}=\int_{R}^{\tau} \tau \partial_{z} a d \tau
$$

Both $A_{\mathrm{t}}^{z}$ and its derivatives vanish on the initial surface $\tau=R$. It is non-zero only because it too does not satisfy the Klein-Gordon equation; rather

$$
\partial^{2} A_{\mathrm{t}}^{z}+e^{2} \eta^{2}\left(A_{\mathrm{t}}^{z}-\frac{2 \theta_{0}}{e} \delta(z)\right)=0
$$

One effect of the extra terms in the field equations is that at late times, $\theta_{\mathrm{t}}$, unlike $\theta_{\mathrm{a}}$ does not become uniform; it tends to the static solution

$$
\lim _{t \rightarrow \infty} \theta_{\mathrm{t}}=\theta_{0} \epsilon(z)\left(1-e^{-e \eta|z|}\right)
$$

Similarly,

$$
\lim _{t \rightarrow \infty} A_{\mathrm{t}}^{z}=\eta \theta_{0} e^{-e \eta|z|}
$$

In a sense phase equilibration never occurs in this gauge. Of course, $D_{z} \theta_{\mathrm{t}}$ does vanish.

Clearly we have to be careful if we use the temporal gauge to allow for these extra terms in the equations of motion. Note that the time derivatives of $\theta_{\mathrm{t}}$ and $A_{\mathrm{t}}^{z}$ do obey the Klein-Gordon equation.

It is also interesting to examine the form of the gauge fields. We easily find that the only non-vanishing components of $F^{\mu \nu}$ are

$$
F^{\alpha z}=x^{\alpha} \partial_{z} a(\tau, z)
$$

In other words, we have a longitudinal electric field, $E^{z}=-t \partial_{z} a$ and azimuthal magnetic field $B^{\phi}=\rho \partial_{z} a$ (in cylindrical polars).

\section{EQUATIONS WITH DISSIPATION}

We now add an extra term to Maxwell's equations:

$$
\partial_{\lambda} F^{\lambda \mu}=j^{\mu}+j_{\mathrm{c}}^{\mu}
$$

where $j_{\mathrm{c}}^{\mu}$ is the conduction current. This represents the effect of dissipation due to coupling with other fields. We shall assume that it can be written in the standard ohmic form, i.e., that the spatial part of $j_{\mathrm{c}}^{\mu}$ is given by

$$
\mathbf{j}_{\mathrm{c}}=\sigma \mathbf{E}
$$


for some suitably defined conductivity $\sigma$. The corresponding charge density $\rho_{\mathrm{c}}$ is fixed by the requirement that the continuity equation,

$$
\dot{\rho}_{\mathrm{c}}+\nabla \cdot \mathbf{j}_{\mathrm{c}}=0
$$

hold (together with the intial condition $\rho_{\mathrm{c}}=0$ at early times). In phase transitions with weak supercooling, bubbles expand on a background of a dense relativistic plasma which is highly conductive. Even in the case of strong supercooling, the energy released in the collision region creates a plasma of its own, and again the conductivity is expected to be high. We shall see that phase equilibration between colliding bubbles is strongly affected by plasma dissipation.

Setting $A^{\mu}=(\phi, \mathbf{A})$, we may write the equations (32) as

$$
-\nabla^{2} \phi-\nabla \cdot \dot{\mathbf{A}}=-e X^{2}(\dot{\theta}+e \phi)+\rho_{\mathrm{c}}
$$

and

$$
\ddot{\mathbf{A}}-\nabla^{2} \mathbf{A}+\nabla \nabla \cdot \mathbf{A}+\nabla \dot{\phi}=e X^{2}(\nabla \theta-e \mathbf{A})-\sigma(\dot{\mathbf{A}}+\nabla \phi) .
$$

The first of these only serves to define $\rho_{\mathrm{c}}$ and is therefore not very useful.

We should really also include a damping term in the equation for $X$. However, we shall not consider that explicitly. Instead, we assume that the oscillations in the radial direction in field space are efficiently damped so that when the bubbles collide $X$ rapidly settles down to its equilibrium value, $X=\eta$.

Let us then specialize to the region inside the bubbles where $X=\eta$. In this case, it seems best to consider first the unitary gauge. (For the moment we drop the subscript u.) The equations then become

$$
\dot{\phi}+\nabla \cdot \mathbf{A}=0
$$

and

$$
\ddot{\mathbf{A}}-\nabla^{2} \mathbf{A}+\sigma \dot{\mathbf{A}}+e^{2} \eta^{2} \mathbf{A}=-\sigma \nabla \phi
$$

If the fields vanish outside a finite volume, we can unambiguously separate the longitudinal and transverse parts of $\mathbf{A}$, writing

$$
\mathbf{A}=\mathbf{A}^{\mathrm{T}}+\nabla \frac{1}{\nabla^{2}} \nabla \cdot \mathbf{A} .
$$

The transverse part satisfies the damped Klein-Gordon equation with mass e $\eta$ :

$$
\ddot{\mathbf{A}}^{\mathrm{T}}-\nabla^{2} \mathbf{A}^{\mathrm{T}}+\sigma \dot{\mathbf{A}}^{\mathrm{T}}+e^{2} \eta^{2} \mathbf{A}^{\mathrm{T}}=\mathbf{0} .
$$

If we look for modes proportional to

$$
e^{p t+i \mathbf{k} \cdot \mathbf{x}},
$$

we find for $p$ the dispersion equation 


$$
p=-\frac{\sigma}{2} \pm i\left(\mathbf{k}^{2}+e^{2} \eta^{2}-\frac{\sigma^{2}}{4}\right)^{1 / 2}
$$

representing a damped oscillation.

For the longitudinal modes, we find the third-order equation

$$
\dddot{\phi}-\nabla^{2} \dot{\phi}+\sigma \ddot{\phi}+e^{2} \eta^{2} \dot{\phi}-\sigma \nabla^{2} \phi=0
$$

which yields

$$
\left(p^{2}+\mathbf{k}^{2}+e^{2} \eta^{2}\right) p+\sigma\left(p^{2}+\mathbf{k}^{2}\right)=0 .
$$

If $\sigma$ is small, there is one real root,

$$
p=p_{1} \approx-\frac{\sigma \mathbf{k}^{2}}{\omega^{2}}
$$

where

$$
\omega^{2}=\mathbf{k}^{2}+e^{2} \eta^{2}
$$

and a complex conjugate pair,

$$
p=p_{ \pm} \approx \pm i \omega-\frac{\sigma e^{2} \eta^{2}}{2 \omega^{2}}
$$

For small $\mathbf{k}$, the latter two modes behave exactly like the transverse modes, but the first one is a much more slowly decaying mode with no oscillation.

On the other hand, if $\sigma \gg e \eta$ and $k \ll e^{2} \eta^{2} / \sigma$, then all three roots are real:

$$
p_{1} \approx-\frac{\sigma k^{2}}{e^{2} \eta^{2}}, \quad p_{2} \approx-\frac{e^{2} \eta^{2}}{\sigma}+\frac{\sigma k^{2}}{e^{2} \eta^{2}}, \quad p_{3} \approx-\sigma+\frac{e^{2} \eta^{2}}{\sigma} .
$$

It should be noted that the slowly-decaying mode $p_{1}$ becomes pure gauge in the limit $k \rightarrow 0$. In the next section we shall see that this mode does not affect the evolution of the gaugeinvariant phase difference $\Delta \theta$.

Now let us briefly consider the equations in the temporal gauge. With $\phi=0$, the equations become

$$
\ddot{\theta}_{\mathrm{t}}-\nabla^{2} \theta_{\mathrm{t}}-e \nabla \cdot \mathbf{A}_{\mathrm{t}}=0
$$

and

$$
\ddot{\mathbf{A}}_{\mathrm{t}}-\nabla^{2} \mathbf{A}_{\mathrm{t}}+\nabla \nabla \cdot \mathbf{A}_{\mathrm{t}}+\sigma \dot{\mathbf{A}}_{\mathrm{t}}+e^{2} \eta^{2} \mathbf{A}_{\mathrm{t}}=-e \eta^{2} \nabla \theta_{\mathrm{t}} .
$$

Of course the equation for the transverse part of $\mathbf{A}_{t}$ is exactly the same as before, eq. (40), but this time if we eliminate $\nabla \cdot \mathbf{A}_{\mathrm{t}}$ using (49) we find for $\theta_{\mathrm{t}}$ a fourth-order equation: in fact $\dot{\theta}_{\mathrm{t}}$ satisfies the same equation (13) as does $\phi_{\mathrm{u}}$. This is actually obvious because the gauge transformation between the two gauges shows that $\dot{\theta}_{\mathrm{t}}=e \phi_{\mathrm{u}}$. 
In the temporal gauge we always have the freedom of making an arbitrary timeindenpendent gauge transformation. Thus the equations have static solutions in which $\theta$ is any arbitrary function; of course $\mathbf{A}$ must be chosen to make $\nabla \theta-e \mathbf{A}=\mathbf{0}$. Note that since only $\dot{\theta}$ appears in the equation, the extra terms discussed in the preceding section do not appear; they would reappear, however, if we tried to write an equation for $\theta$ itself, incorporating the chosen initial conditions. In a non-simply connected region, the $\theta_{\mathrm{t}}$ equation allows more general types of solution, in which it is not single-valued. For example, we could mimic a closed loop by imposing periodic boundary conditions, and look for solutions with $\theta(z+L)=\theta(z)+2 n \pi$. One such is $\theta=2 n \pi z / L$, with $A^{z}=2 n \pi / e L$. This is similar to the type of solution one would expect to see eventually around a string, once the oscillations have died down.

\section{COLLISIONS BETWEEN PLANAR WALLS}

As in Section II, we shall first consider the central region of a two-bubble collision, where the problem reduces to one-dimensional form.

In one dimension of course the only modes are longitudinal, so we should use (43) or its equivalent in the temporal gauge. We have assumed that $X$ settles down rapidly to its equilibrium value. It is reasonable then to take as our initial condition, just after the bubbles have collided, say at $z=0$ at $t=0$, a configuration where $X=\eta$ and (in the temporal or axial gauge) $\theta$ has a discontinuous jump from $-\theta_{0}$ to $\theta_{0}$, as in (12). We also assume that at that time there are no electromagnetic fields present, so we may take

$$
\mathbf{A}_{\mathrm{t}}(0, z)=\mathbf{0}, \quad \dot{\mathbf{A}}_{\mathrm{t}}(0, z)=0 .
$$

We can now transform back to the unitary gauge, where we find as before the initial conditions

$$
\begin{gathered}
\phi(0, z)=0, \quad \dot{\phi}(0, z)=\frac{2 \theta_{0}}{e} \delta^{\prime}(z), \\
A^{z}(0, z)=-\frac{2 \theta_{0}}{e} \delta(z), \quad \dot{A}^{z}=0 .
\end{gathered}
$$

The solution is immediate:

$$
\phi(t, z)=\int \frac{d k}{2 \pi} e^{i k z}\left(\varphi_{1} e^{p_{1} t}+\varphi_{+} e^{p_{+} t}+\varphi_{-} e^{p_{-} t}\right)
$$

where $p_{1}$ and $p_{ \pm}$are given by (45) and (47) and the amplitudes $\varphi_{1}, \varphi_{ \pm}$satisfy

$$
\begin{aligned}
\varphi_{1}+\varphi_{+}+\varphi_{-} & =0 \\
p_{1} \varphi_{1}+p_{+} \varphi_{+}+p_{-} \varphi_{-} & =2 i k \theta_{0} / e \\
p_{1}^{2} \varphi_{1}+p_{+}^{2} \varphi_{+}+p_{-}^{2} \varphi_{-} & =0
\end{aligned}
$$

The solution is 


$$
\varphi_{1}=-\frac{2 i k \theta_{0}}{e} \frac{p_{+}+p_{-}}{\left(p_{+}-p_{1}\right)\left(p_{-}-p_{1}\right)},
$$

with two similar equations obtained by permutation of $(1,+,-)$.

The gauge-invariant phase difference (4) can be written as

$$
\Delta \theta \equiv \int_{-\infty}^{\infty} D_{z} \theta d z=-e \int_{-\infty}^{\infty} A_{\mathrm{u}}^{z} d z
$$

and can be evaluated using Eq.(54). Alternatively, we can obtain an equation for $\Delta \theta(t)$ by integrating (36),

$$
\left(\frac{d^{2}}{d t^{2}}+\sigma \frac{d}{d t}+e^{2} \eta^{2}\right) \Delta \theta=0
$$

The boundary conditions corresponding to (53) are

$$
\Delta \theta(0)=2 \theta_{0}, \quad \Delta \dot{\theta}(0)=0,
$$

and the solution is

$$
\Delta \theta(t)=A_{+} e^{p_{+} t}+A_{-} e^{p_{-} t}
$$

where

$$
p_{ \pm}=-\frac{\sigma}{2} \pm i\left(e^{2} \eta^{2}-\frac{\sigma^{2}}{4}\right)^{1 / 2}
$$

and

$$
A_{ \pm}=\mp \frac{2 \theta_{0} p_{\mp}}{p_{+}-p_{-}} .
$$

Note that the values of $p_{ \pm}$are the same as in Eq.(42) for the transverse modes at $k=0$.

In the small- $\sigma$ case, we find

$$
\Delta \theta(t)=2 \theta_{0} e^{-\sigma t / 2}\left(\cos e \eta t+\frac{\sigma}{2 e \eta} \sin e \eta t\right) .
$$

It is interesting to compare this with the corresponding expression, (17), in the undamped case. The oscillating factor is essentially the same in both cases. The difference is that here the dissipation causes exponential damping, while in the previous case the spherical geometry led to a linear damping. For large $\sigma$ there is no oscillation, and Eqs.(60)-(62) give

$$
\Delta \theta(t)=2 \theta_{0} \exp \left(-e^{2} \eta^{2} t / \sigma\right)
$$

The characteristic time scale for the current damping, $t_{d}$, is given by

$$
t_{d} \sim 1 / \sigma \quad(\sigma \lesssim e \eta), \quad t_{d} \sim \sigma / e \eta \quad(\sigma \geq e \eta)
$$


It is also interesting to look at the solution directly in the temporal gauge. There, we have to solve a fourth-order equation, so in addition to (12) we need initial conditions for $\ddot{\theta}$ and $\dddot{\theta}$. These are given by (51) together with (49):

$$
\ddot{\theta}(0, z)=2 \theta_{0} \delta^{\prime}(z), \quad \dddot{\theta}(0, z)=0 .
$$

Thus we find

$$
\theta_{\mathrm{t}}(t, z)=\int \frac{d k}{2 \pi} e^{i k z}\left(\vartheta_{0}+\vartheta_{1} e^{p_{1} t}+\vartheta_{+} e^{p_{+} t}+\vartheta_{-} e^{p_{-} t}\right)
$$

It is easy to verify, however, that in fact $\vartheta_{0}$ vanishes; for small $\sigma$, we have

$$
\vartheta_{0}=0, \quad \vartheta_{1} \approx-\frac{2 i \theta_{0} e^{2} \eta^{2}}{k \omega^{2}}, \quad \vartheta_{ \pm} \approx-\frac{i k \theta_{0}}{\omega^{2}}\left(1 \mp \frac{3 i e^{2} \eta^{2} \sigma}{2 \omega^{3}}\right)
$$

It is interesting to note that in the limit $k \rightarrow 0, \vartheta_{ \pm} \rightarrow 0$ while $\vartheta_{1} \sim-2 i \theta_{0} / k$. This shows that as before

$$
\lim _{z \rightarrow \pm \infty} \theta_{\mathrm{t}}= \pm \theta_{0}
$$

although $\theta_{\mathrm{t}}(t, z)$ approaches its limit more slowly at late times.

We can also find a solution corresponding to an idealized one-dimensional version of a three-bubble collision, by imposing periodic boundary conditions. We may represent the three bubbles at the moment when they meet by the segments $\left[0, L_{1}\right],\left[L_{1}, L_{2}\right]$ and $\left[L_{2}, L\right]$ of the $z$ axis. If we choose the initial phases to be $0, \theta_{1}, \theta_{2}$, where $\theta_{1}, \theta_{2}-\theta_{1}$ and $2 \pi-\theta_{2}$ all lie between 0 and $\pi$, then it is natural to impose the boundary condition $\theta_{\mathrm{t}}(L)=\theta_{\mathrm{t}}(0)+2 \pi$. In the unitary gauge, the initial conditions for $\dot{\phi}$, as in (52), become

$$
\dot{\phi}(0, z)=\frac{2 \theta_{1}}{e} \delta^{\prime}\left(z-L_{1}\right)+\frac{2\left(\theta_{2}-\theta_{1}\right)}{e} \delta^{\prime}\left(z-L_{2}\right)+\frac{2\left(2 \pi-\theta_{2}\right)}{e} \delta^{\prime}(z) .
$$

The solution is similar to (54) but with the Fourier integral replaced by a sum over the modes $k_{n}=2 n \pi / L$, and the right hand side of (55) replaced by a sum of three terms with appropriate phases. An interesting special case is the one where $L_{1}=L / 3, L_{2}=2 L / 3$, while $\theta_{1}=2 \pi / 3$ and $\theta_{2}=4 \pi / 3$. Then only modes with $n \equiv 0 \quad(\bmod 3)$ will contribute. The non-vanishing coefficients $\varphi_{0,3 n}$ and $\varphi_{ \pm, 3 n}$ are of essentially the same form as in (56). Note that there is no $n=0$ term. The boundary condition on $\theta_{\mathrm{t}}$ must be accommodated by adding to the Fourier series an extra constant term, for example the linear term $2 \pi z / L$.

\section{MAGNETIC FLUX}

Let us now move away from the immediate neighbourhood of the point of first contact between the bubbles. In particular, we want to examine the magnetic fields present in the region where the collision occurs.

For simplicity, we again suppose that the two colliding bubbles have equal radii, $R$, at the moment of collision. The main effect of the curvature is to delay the collision at points off the line of centres. If the collision occurs at the origin at time $t=0$, then at a transverse 
distance $x$, it will occur (to first order in the curvature) at time $t=x^{2} / 2 R v$, where $v$ is the velocity of the bubble walls. As a first approximation, we may expect that the fields near $x=y=0$ have the same form as in the planar case, while more generally, for example,

$$
\theta(t, x, y, z) \approx \theta\left(t-\frac{x^{2}+y^{2}}{2 R v}, 0,0, z\right) .
$$

This of course has the effect of introducing transverse gradients into the fields. Consequently, $j^{x}$ and $j^{y}$ are non-zero and in turn generate transverse components of $\mathbf{A}$. The physical result is the appearance (as in the undamped case) of an azimuthal magnetic field. The magnetic flux actually forms a loop around the region where the collision is occurring.

We are particularly interested in the total magnetic flux. It is possible to calculate that without going into the details, by looking at the line integral of the vector potential.

Consider a rectangular loop $A B C D$, where (see Figure 1)

$$
A, B=(0,0, \mp Z), \quad C, D=(X, 0, \pm Z) .
$$

The values of $X$ and $Z$ are chosen so that during the time interval of interest, all four points are within the bubbles, but the path $C D$ passes outside them. We have assumed that there are no fields outside the bubbles. For simplicity, let us suppose that $\Phi$ remains slightly nonzero, so that the phase $\theta$ is well defined and interpolates smoothly between its values inside the bubbles. (It would of course make no difference if we assumed some other distribution, so long as $\Phi$ remains very small, but the argument is simpler in this case.) It is easy to see that initially, when the bubbles have just met,

$$
\oint_{A B C D} D_{k} \theta d x^{k}=0 .
$$

In fact, using the definition (57), one sees that $\Delta \theta_{A B}=\Delta \theta_{D C}=2 \theta_{0}$, while $\Delta \theta_{B C}=\Delta \theta_{D A}=$ 0 . On the other hand, after a time $t \gg t_{d}$, with $t_{d}$ given by (65), dissipation has reduced $\Delta \theta_{A B}$ to zero, while leaving the other three legs unaltered. Thus

$$
\oint_{A B C D} D_{k} \theta d x^{k}=-2 \theta_{0} .
$$

By hypothesis the loop contains no zeros of $\Phi$, so $\theta$ is single-valued. Thus it follows at once that

$$
\int_{-Z}^{Z} d z \int_{0}^{X} d x B^{y}=\oint_{A B C D} A_{k} d x^{k}=\frac{2 \theta_{0}}{e} .
$$

This is exactly what we should expect. Note that when three bubbles meet the three magnetic flux tubes combine. If there is a total net phase change of $2 \pi$ around the loop, the total flux trapped is exactly one flux quantum, $2 \pi / e$.

It is interesting in passing to see how the result (74) emerges in different gauges. In the unitary gauge, of course, each $\Delta \theta$ is given by a line integral of $\mathbf{A}$; the essential effect is that along $A B, A^{z}$ is non-zero initially, but zero finally. In the temporal gauge, the situation is reversed. There is no change in the values of $\theta$ at the four corners. Initially, $A^{z}$ vanishes along $A B$, but after the dissipation its line integral exactly cancels $\theta_{B}-\theta_{A}$. The most complicated situation occurs in the axial gauge. Here we find that finally $\theta_{A}=\theta_{B}=0$, which ensures that $\Delta \theta_{A B}$ vanishes. However, to keep the line integrals along the transverse sections $B C$ and $D A$ zero, $A^{x}$ must acquire a non-zero value to compensate for the transverse gradient of $\theta$; it is easy to check that it does. 


\section{FLUX SPREADING}

At any particular time $t$ after the initial collision, the magnetic flux forms a ring. We can readily estimate its size. The outer edge occurs where the bubbles are just meeting, namely at a transverse distance

$$
x=\sqrt{2 R v t} .
$$

The time taken for the currents generated to be dissipated at any given point is $t_{d}$ given by Eq.(65). The inner edge of the ring occurs where dissipation is essentially over, namely at

$$
x=\sqrt{2 R v\left(t-t_{d}\right)}
$$

Note that the ring becomes narrower as it expands. This is because it is slowing down. The speed of expansion is $\sqrt{R v / 2 t}$. If $v=c$, this is always superluminal (the small-angle approximation breaks down of course when $t$ becomes of order $R$ ). In that regime, one should not think of a tube of magnetic flux moving outwards, but rather of magnetic flux being created and destroyed at successively larger radii. On the other hand, if $v \ll c$, the speed may eventually be less than $c$. At that point, we can no longer disregard the region outside the bubbles.

While the magnetic field is expelled from bubble interiors (as in the Meissner effect), it has a tendency to spread at the speed of light in the false vacuum region outside the bubbles. If the conductivity due to particles present in that region can be neglected, then the outer radius of the magnetic flux ring is $x \sim t$, while the inner radius is given by Eq.(76). At $t>R$ most of the flux will escape to distances greater than the bubble radii. If a third bubble arrives after that, the probability of vortex formation in this three-bubble collision will be strongly suppressed.

Realistically, however, the case of low bubble velocities corresponds to weak supercooling, when bubbles expand on a background of relatively dense plasma, and the conductivity in the exterior region is far from being negligible. The magnetic field dynamics in a conducting medium is described by the equation

$$
\ddot{\mathbf{B}}-\nabla^{2} \mathbf{B}+\sigma \dot{\mathbf{B}}=0 .
$$

It is easily seen that the characteristic time of magnetic field variation on a length scale $L$ is $t \sim \sigma L^{2}$, and thus the speed of magnetic flux spreading is

$$
v_{B} \sim L / t \sim(\sigma L)^{-1}
$$

In the limit of very high conductivity, $v_{B} \rightarrow 0$ (the magnetic flux is "frozen" into the plasma). If the typical bubble radius at collision is $\xi$ and the velocity is $v$, then the condition for the flux spreading to be negligible is

$$
\sigma \xi v \gg 1
$$

In order to assess the validity of this condition, we shall need estimates for $\xi, \sigma$ and $v$. The radius $\xi$ can be estimated as $\xi \sim(v / \gamma)^{1 / 4}$, where $\gamma$ is the bubble nucleation rate. To estimate the conductivity $\sigma$, we represent the drift velocity of charged particles as 


$$
v_{d} \sim p / \epsilon \sim e E \tau / T
$$

where $p$ is the average momentum gained by a particle between collisions, $E$ is the electric field, $\tau$ is the mean free time, $T$ is the temperature, and $\epsilon \sim T$ is the average particle energy. The mean free time is $\tau \sim(n \tilde{\sigma})^{-1}$, where $\tilde{\sigma} \sim e^{4} / T^{2}$ is the characteristic scattering crosssection and $n$ is the density of charged particles. Now, the current density is $j \sim n e v_{d} \sim$ $\left(T / e^{2}\right) E$, and thus

$$
\sigma \sim T / e^{2}
$$

and the condition (80) can be rewritten as

$$
v \gg e^{2} / \xi T \text {. }
$$

Since the average bubble separation $\xi$ is expected to be greater than the thermal particle wavelength $T^{-1}$, the right-hand side of (83) does not exceed $10^{-2}$. The temperature at the phase transition is typically $T \sim \eta$, and Eq. (82) corresponds to the high-conductivity regime, $\sigma \gg e \eta$.

The bubble velocity $v$ in the overdamped regime can be roughly estimated from $f \sim \Delta \rho_{v}$, where $f$ is the force of friction due to particle scattering off the bubble wall, and $\Delta \rho_{v}$ is the vacuum energy difference between the two sides of the wall. Both $f$ and $\Delta \rho_{v}$ are modeldependent, but in all cases $f \lesssim T^{4} v$, which gives a lower bound on $v$,

$$
v \gtrsim \Delta \rho_{v} / T^{4}
$$

Typically, one finds that bubble velocities are rather high. For example, a detailed analysis for the case of electroweak phase transition gives $v \sim 0.1-1$. This suggests that the condition (83) is typically satisfied, so that the magnetic flux does not escape. Exceptions may occur in cases of nearly degenerate vacua, when $\Delta \rho_{v}$ is very small.

\section{CONCLUSIONS}

Our main conclusions can be formulated in terms of the gauge-invariant phase difference $\Delta \theta$ defined in Eq.(4). We found that $\Delta \theta$ remains equal to its initial value while the bubbles are well-separated and undergoes damped oscillations after the collision. The oscillation period is determined by the gauge boson mass, and the damping time by the plasma conductivity. Both timescales are typically much smaller than the bubble radii at the time of collision. This is to be contrasted to the case of a global symmetry breaking, when the phases in the bubble interiors can change only after the arrival of the Goldstone boson waves propagating at the speed of light from the collision region.

The currents associated with the oscillating phase gradients generate a magnetic field which is concentrated in a ring-shaped region near the circle on which the bubble walls intersect. The total magnetic flux is determined by the initial value of $\Delta \theta$,

$$
\Phi_{B}=\Delta \theta_{0} / e
$$

Right after the collision, the radius of the intersection circle grows faster than the speed of light, but if the bubble expansion is substantially slowed down by interaction with ambient 
plasma, the speed may eventually become nonrelativistic. The magnetic field then begins to spread into the exterior region and can in principle escape to distances large compared to the bubble radii. However, magnetic field has a tendency to be "frozen" into a conducting plasma, and the speed of spreading is typically smaller than the bubble expansion speed (with the exception of cases of nearly degenerate vacua, when the bubble velocities are extremely small).

In a three-bubble collision, the three magnetic flux tubes combine when the bubbles meet. The resulting magnetic flux is $\Phi_{B}=\Delta \theta_{\text {tot }} / e$, where $\Delta \theta_{\text {tot }}=2 \pi n$ is the sum of the initial phase differences between the bubbles and $n$ is an integer. For $n \neq 0$, a vortex is formed carrying $n$ flux quanta, $\Phi_{B}=2 \pi n / e$. Thus, although the physics of bubble collisions turned out to be interesting and complicated, our conclusions are essentially the same as those resulting from the naive analysis in Ref. [3].

In conclusion, we would like to mention some open questions. As a matter of principle, it would be interesting to understand what happens when the bubble expansion speed is so low that most of the magnetic flux does escape. One expects that the vortex density in this case is suppressed, and we are currently investigating what effect this may have on the properties of the resulting string distriution.

Throughout the paper we assumed that the radial mode of the Higgs field is strongly damped and settles to its equilibrium value on a timescale short compared to the phase equilibration process. In general, however, the energy dissipation in the radial mode is itself a complicated process and can take considerable time, especially in the case when the colliding walls are relativistic. The phase equilibration in this case can be studied using numerical simulations.

Another interesting and important question is what effect thermal fluctuations of the magnetic field and of winding number have on the vortex formation. A naive estimate of the magnetic flux fluctuation on scale $\xi$ is $\delta \Phi_{B} \sim(\xi T)^{1 / 2}$, where $T$ is the temperature. This could in principle be much greater than a single flux quantum. If all this flux is squeezed into a vortex by advancing bubble walls, then we can expect that a sizable proportion of vortices will have large winding numbers. A particularly interesting situation may arise in models where the gauge bosons are heavier than the Higgs particles, so that $n>1$ strings are stable. In this case one expects the formation of an interconnected network of strings with different winding numbers. The cosmological evolution of such a network may be very different from the standard picture.

Thermal fluctuations may also play an important role in defect formation during firstorder phase transitions with a global symmetry breaking. In this case, the phase $\theta$ is a massless Goldstone field, and its fluctuation on scale $\xi$ is $\delta \theta \sim(T \xi)^{1 / 2}$. Once again, this can be large, resulting in the formation of large vortex clusters. We note, however, that multiply charged vortices are unstable in the case of a global symmetry breaking, and we expect the clusters to disperse.

\section{ACKNOWLEDGMENTS}

We are indebted for hospitality to the Isaac Newton Institute for Mathematical Sciences, Cambridge, England, where this work was begun. We have benefited from discussions with 
numerous participants, in particular Allen Everett, Eugene Chudnovsky, Mark Hindmarsh, Tanmay Vachaspati and Julian Borrill. 


\section{REFERENCES}

[1] For recent reviews, see A. Vilenkin \& E.P.S. Shellard, Cosmic Strings and other Topological Defects (Cambridge: Cambridge University Press, 1994); M.B. Hindmarsh \& T.W.B. Kibble, Cosmic Strings, submitted to Rep. Prog. Phys. (hep-ph/9411342).

[2] S. Coleman, Phys. Rev. D15, 2929 (1977).

[3] T.W.B. Kibble, J. Phys. A: Math. \& Gen. 9, 1387 (1976).

[4] T. Vachaspati, Phys. Rev. D44, 3723 (1991).

[5] S. Rudaz \& A.M. Srivastava, Mod. Phys. Lett. A8, 1443 (1993).

[6] M. Hindmarsh, A.-C. Davis \& R. Brandenberger, Phys. Rev. D49, 1944 (1994).

[7] S.W. Hawking, I.G. Moss, and J.M. Stewart, Phys. Rev. D26, 2681 (1982). 


\section{FIGURES}

FIG. 1.

Two colliding bubbles, showing the closed path $A B C D$. 
This figure "fig1-1.png" is available in "png" format from: http://arxiv.org/ps/hep-ph/9501266v1 